\title{
APROXIMACIÓN AL TURISMO RELACIONADO CON LAS ZONAS DE BAÑO EN EXTREMADURA: UN META-ANÁLISIS A PARTIR DE TRABAJOS ACADÉMICOS INÉDITOS
}

\author{
Manuel Pulido Fernández* \\ Universidad de Extremadura \\ https://orcid.org/0000-0001-9340-0107 \\ Rafael Robina Ramírez** \\ Universidad de Extremadura \\ https://orcid.org/0000-0002-7117-8645 \\ Jacinto Garrido Velarde*** \\ Universidad de Extremadura \\ https://orcid.org/0000-0002-1404-1255
}

\section{RESUMEN}

Más de un centenar de trabajos académicos son presentados anualmente en las facultades españolas con el objetivo de obtener un título de Graduado en Turismo. Muchos de ellos, se mantienen inéditos, a pesar de la utilidad de los datos que recopilan, bien por aspectos formales o por la escasa envergadura que presentan en sí de manera aislada. En este trabajo, por tanto, se han recopilado y analizado los datos de una serie de estudios que han sido llevados a cabo por estudiantes de turismo, y cuyo objetivo era analizar la oferta y la demanda turística de zonas de baño de Extremadura con diferentes características: embalses, gargantas, balnearios y una antigua cantera. Todas ellas comparten fortalezas como la pertenencia a espacios protegidos y la cercanía a numerosos recursos turísticos pero se observan diferentes aprovechamientos en términos de oferta hotelera y de restauración. Respecto a la demanda, predomina el turismo nacional, con vínculos a las localidades cercanas y con una mayor concentración en los fines de semana.

Fecha de recepción: 20 de diciembre de 2019.

Fecha de aceptación: 15 de mayo de 2020.

* Facultad de Filosofía y Letras. Universidad de Extremadura. Avenida de la Universidad, s/n. 10071 CÁCERES (España).E-mail: mapulidof@unex.es

** Facultad de Empresa, Finanzas y Turismo. Universidad de Extremadura. Avenida de la Universidad, s/n. 10071 CÁCERES (España). E-mail: rrobina@unex.es

*** Facultad de Educación. Universidad de Extremadura. Avenida de Elvas, s/n. 06006 BADAJOZ (España). E-mail: jgvelarde@unex.es 
Palabras clave: Turismo estival; embalses; gargantas; recursos turísticos; Trabajos académicos.

\title{
Approach to tourism related to bathing areas in Extremadura: a meta-analysis based on unpublished students' works
}

\begin{abstract}
More than a hundred academic works are presented annually in Spanish faculties with the aim of obtaining a degree in Tourism. Many of them remain unpublished, despite the usefulness of their data, either because formal aspects or the limited size of their studies. In this work, we have collected and analyzed a sort of academic works in which tourist supply and demand of bathing areas of Extremadura (reservoirs, gorges and an old quarry) have been the core of the study. All of them share strengths such as belonging to protected areas and the proximity to numerous tourist resources but different uses are observed in terms of hotel and restaurant offer. Regarding demand, national tourism linked to nearby towns with a greater concentration on weekends is predominant.
\end{abstract}

Keywords: Summer tourism; reservoirs; gorges; tourism resources; Academic works.

\section{INTRODUCCIÓN}

La llegada de turistas ha crecido un 55\% desde 2010, hasta alcanzar los 82 millones de visitantes en 2017, posicionando al turismo exterior como uno de los principales motores de la economía española durante la recuperación. Sin embargo, el impulso del turismo extranjero se está ralentizando desde 2018 (Diez et al., 2019) y las restricciones de movilidad causadas por la pandemia del COVID-19 de 2020 auguran una mayor relevancia de los movimientos intranacionales, al menos, en los próximos meses.

La potencialidad de las aguas de interior en España como reclamo turístico se ha hecho especialmente patente en este verano de 2020 debido a las restricciones de acceso al turismo internacional, mayoritariamente consumidor de sol y playa. No obstante, autores como, por ejemplo, López Olivares (2001) o Cebrián Abellán y García González (2010), entre otros muchos, ya han constatado el atractivo turístico de los recursos del interior (incluyendo sus aguas dulces) de provincias como Alicante o Albacete, normalmente obnubilado por el interés que suscitan localidades costeras como Denia, Jávea, Altea, Calpe, Guardamar del Segura, etc.

En Extremadura, con un mercado turístico marcadamente nacional, el turismo representa apenas poco más del 5\% del Producto Interior Bruto (PIB), sin embargo, se ha observado en los últimos años una tendencia al alza, al menos en el número de turistas y pernoctaciones de los visitantes (según los datos publicados periódicamente por el Observatorio Turístico de Extremadura), que arrojan indicios de resultados positivos como consecuencia, presumiblemente, de las políticas de promoción turística llevadas a cabo en la región, de la puesta en valor de espacios naturales mediante figuras de protección y de la mejora en las infraestructuras de comunicación (construcción de autovías como la A-66 y la A-58) desde finales del siglo XX y principios del XXI (Nieto Masot y Cárdenas Alonso, 2017). 
Una de las muchas razones que pueden estar detrás de este aparente despegue turístico es, por un lado, la apuesta por la diversidad, ya sea en forma de modalidades turísticas o mediante la promoción de diferentes productos turísticos (Dirección General de Turismo, 2010) y, por otro, su valioso patrimonio natural avalado por figuras de protección de carácter internacional, estatal y autonómico. En la actualidad, la región cuenta con 3 Reservas de la Biosfera de la UNESCO (Monfragüe, Tajo Internacional y La Siberia), 1 Geoparque de la Red Global de Geoparques (Villuercas, Ibores, Jara), 2 Humedales de Importancia Internacional, 166 espacios naturales protegidos (ENP) por la Red Natura 2000, 1 Parque Nacional (Monfragüe) y 40 ENP por figuras de protección autonómica (ley 8/1998 de conservación de la naturaleza y de espacios naturales de Extremadura; modificada por la ley 9/2006).

En la última década, diferentes estudios han analizado las posibilidades turísticas de estos atractivos naturales. Por ejemplo, Leco Berrocal et al. (2015) se centraron en la demanda turística de Monfragüe (Parque Nacional desde 2007 y Reserva de la Biosfera desde 2003). Uno de los datos más interesantes que arroja este trabajo es que sólo el 6,7\% de las personas que visitan Monfragüe lo hacen motivados por el hecho de ser Reserva de la Biosfera (RB) de la UNESCO. Algo parecido ha sido observado por Pino-del Carpio et al. (2014) en el resto de RB que conforman la red española, que siguen sin ser un reclamo turístico. La figura de Geoparque, al menos en el caso de Extremadura, sigue siendo poco apreciada, desde un punto turístico, ya que el principal atractivo es el Monasterio de Guadalupe (Robina Ramírez y Pulido Fernández, 2018).

Extremadura, con sus más de $40.000 \mathrm{~km}^{2}$ de superficie, además, almacena en torno al $30 \%$ del agua de origen fluvial que hay en España, gracias a una serie de grandes embalses, con diferentes aprovechamientos (regadío, hidroeléctrico, etc.), construidos a lo largo de los ríos Tajo y Guadiana (y afluentes) durante el siglo XX (Junta de Extremadura, 2016). A eso, hay que añadir, la presencia de sistemas montañosos de relativa importancia, como el Sistema Central y los Montes de Toledo, que la provisionan de una serie de gargantas aptas para el baño, sobre todo en el norte de la región (Abel Schaad et al., 2009), y una geología particular que confiere aptitudes para el termalismo en diferentes puntos de Extremadura (Rebollada Casado et al., 2016).

En términos de turismo de baño, Extremadura es la región con más kilómetros de costa interior (agua dulce) de Europa Occidental. Embalses como los de Alqueva, Alcántara, Gabriel y Galán, Orellana, La Serena o Valdecañas totalizan más de $1.500 \mathrm{~km}$ de costa interior y ocupan el 1,75\% de la superficie total de la región (Andrades Caldito, 2008). No obstante, esta cifra debe ser tomada con cautela, ya que gran parte de esos $1.500 \mathrm{~km}$ de costa no tienen ninguna potencialidad para ser aprovechados como zonas de baño. En total, se contabilizan 72 piscinas naturales (playas fluviales y gargantas), de las cuales 2 cuentan con el distintivo de bandera azul (Orellana y Cheles), a lo que hay que sumar que la gran mayoría de municipios cuentan, al menos, con piscina municipal (Alonso de la Torre, 2019).

A pesar de la importancia que estas zonas de baño tienen como factor de atracción para los turistas en los meses de verano, apenas existen trabajos publicados sobre la temática en la región. García González (1991; 2004), por ejemplo, es uno de los pocos que ha abordado, aunque desde un enfoque muy genérico, la relación entre agua y turismo en Extremadura. Otros trabajos pueden ser consultados en Internet o han sido mencionados de 
manera pasajera en congresos de la temática o en reuniones de carácter académico, pero se echa de menos la existencia de trabajos profundos que abarquen esta temática (Campesino Fernández, 2016). Mención aparte merecen aquellos trabajos que se han centrado, sobre todo, en el patrimonio cultural pasado y presente, ligado a la construcción de embalses y otras infraestructuras hídricas e hidráulicas (Sánchez Lomba, 2014) y que, en algunos casos, incluso se encuentra sumergido bajo las aguas de algún gran embalse (Matamoros Coder y Carrascosa Moliner, 2014).

Por esta razón, una serie de profesores de la Facultad de Empresa, Finanzas y Turismo de la Universidad de Extremadura, tomamos la decisión de intentar abordar esta temática de estudio, al menos, inicialmente mediante trabajos académicos, hasta que se puedan articular proyectos de investigación de carácter más profundo. Fruto de esta iniciativa, iniciada en 2016, se han conseguido defender públicamente una serie de trabajos académicos para la obtención del título de Turismo, en los que se ha podido abordar, sobre todo a escala de enclave, en la indagación de aspectos concernientes a la oferta y demanda turística de una serie de lugares conocidos (regionalmente) como zonas de baño.

Muchos de estos trabajos, permanecen hoy en día como inéditos, ya que la publicación en los repositorios de la Universidad queda reservada como premio a los mejores trabajos. La razón por la que estos trabajos no llegan a obtener la nota necesaria para ser publicados responde, en muchos casos, a aspectos formales motivados por la capacidad intelectual o la inmadurez contextual de cada tutorando y, en otros, a una falta de ambición en el muestreo, ya que, en muchas ocasiones, los autores y los tutores se enfrentan a limitaciones que impiden hacer trabajos con mayor rigor, bien sean por falta de tiempo, recursos, proyectos o líneas de investigación que den soporte a dichos trabajos.

No obstante, y sin tener en cuenta la calidad o nota final que cada estudio haya obtenido por separado, lo que, al final, resulta evidente es que la sociedad o la comunidad científica no podrá disfrutar de una serie de datos valiosos, que permanecerán desaprovechados por el hecho de que sólo han sido consultados por los autores, tutores y miembros del tribunal de cada trabajo y son inaccesibles para el resto de interesados. En este sentido, este trabajo pretende ser también una reivindicación para la publicación de este tipo de información, que representa el esfuerzo puntual de estudiantes y tutores y que, en algunos casos, puede suministrar información proveniente de encuestas, trabajo de campo, y otras metodologías, que hasta la fecha no están siendo abordados por ningún otro tipo de investigación.

Teniendo en cuenta todo esto, este trabajo pretende recopilar y analizar la información publicada en una serie de trabajos académicos de estudiantes, a los que se ha tenido acceso por ser tutores o miembros de tribunal, que abordan la temática de las áreas turísticas vinculadas al baño estival en Extremadura desde la perspectiva de la oferta y la demanda, y a muy diversas escalas, mediante encuestas, cuestionarios y trabajo de campo. Este estudio, por tanto, ha permitido la comparación entre diversas áreas de baño, como, por ejemplo, gargantas del norte de Extremadura o playas fluviales hechas sobre los grandes embalses, trazándose analogías y diferencias entre los enclaves estudiados.

El trabajo se estructura, además de con esta sección introductoria, en un capítulo metodológico, en el que se presentan las áreas de estudio (2.1.) y se describe cómo fue abordada la recopilación y el análisis de datos respecto a los recursos turísticos (2.2.), 
oferta (2.3.) y demanda turística (2.4.), y en un capítulo de resultados donde se presenta la información obtenida siguiendo el mismo orden que en la metodología: 3.1. Recursos turísticos, 3.2. Oferta turística y 3.3. Demanda turística. Finalmente, se añaden un capítulo de discusión (4.) y otro de conclusiones (5.), que sirven para contextualizar los hallazgos aquí encontrados y para definir futuras líneas de investigación, bien sean soportadas por trabajos académicos (trabajos de índole parcial) o líneas lideradas por profesores a través de proyectos de investigación (trabajos más amplios, consistentes e integrales).

\section{MATERIAL Y MÉTODOS}

\section{1. Área de estudio}

Los trabajos aquí recopilados y analizados fueron realizados en 4 áreas de baño: [1] Embalse de Orellana (Orellana, BA) (Vázquez Wizner, 2017), [2] Isla del Zújar (Castuera, BA) (Santos Redondo, 2018), [3] Garganta de los Infiernos (Valle del Jerte, CC) (Gómez Sánchez, 2016) y [4] Cantera de Cabezos (Alcántara, CC) (Fernández Barroso, 2020) (Figura 1).

Al este de Extremadura, en la provincia de Badajoz, se localizan los embalses de Orellana y del Zújar, más concretamente, en los municipios de Orellana la Vieja y Castuera (comarcas de la Siberia y la Serena). Ambos son embalses construidos en el siglo XX sobre los ríos Guadiana y Zújar (afluente del Guadiana) buscando un aprovechamiento agrícola (regadío) e hidroeléctrico. El uso turístico actual, como zonas de baño, se concentra en sus playas fluviales, en torno a las cuales se han generado una serie de instalaciones y servicios turísticos.

La Garganta de los Infiernos se localiza al norte de Extremadura, en la Comarca del Valle del Jerte (localidades de Cabezuela del Valle y Jerte). El tramo de baño por excelencia de este curso fluvial son Los Pilones, en los que predomina una serie de marmitas de gigante, esculpidas sobre un lecho de roca granítica, interconectadas entre sí como si de una secuencia de mini cascadas se tratase. Esta zona es Reserva Natural (figura de protección autonómica) debido al ecosistema característico de curso de agua de alta montaña, típico de las sierras graníticas del Sistema Central.

La Cantera de Cabezos se localiza en la localidad de Alcántara, al este de Extremadura y muy cerca de la frontera con Portugal, al lado del embalse hidroeléctrico del cual sirvió como proveedor de materiales de construcción para su obra. En la actualidad, dicha cantera abierta ex proceso para la construcción del embalse (100 m de profundidad) se llena regularmente de agua de lluvia y de filtraciones subterráneas (manantiales), que la han hecho convertirse en una de las zonas de baño más auténticas de la comarca.

Las cuatro áreas de baño aquí tratadas (descritas en el apartado anterior), junto con otro trabajo de turismo termal (balnearios) a escala autonómica que también ha sido analizado y debatido (Viana Cardador, 2018), han seguido una lógica de trabajo muy similar secuenciada en cuatro fases (algunos trabajos tan solo han llevado a cabo las dos primeras) con respecto a los recursos turísticos: [1] recopilación e identificación, [2] selección y descripción, [3] inventario y catalogación, y [4] puntaje y jerarquización. 


\section{Figura 1 \\ LOCALIZACIÓN GEOGRÁFICA DE LAS ÁREAS DE ESTUDIO}

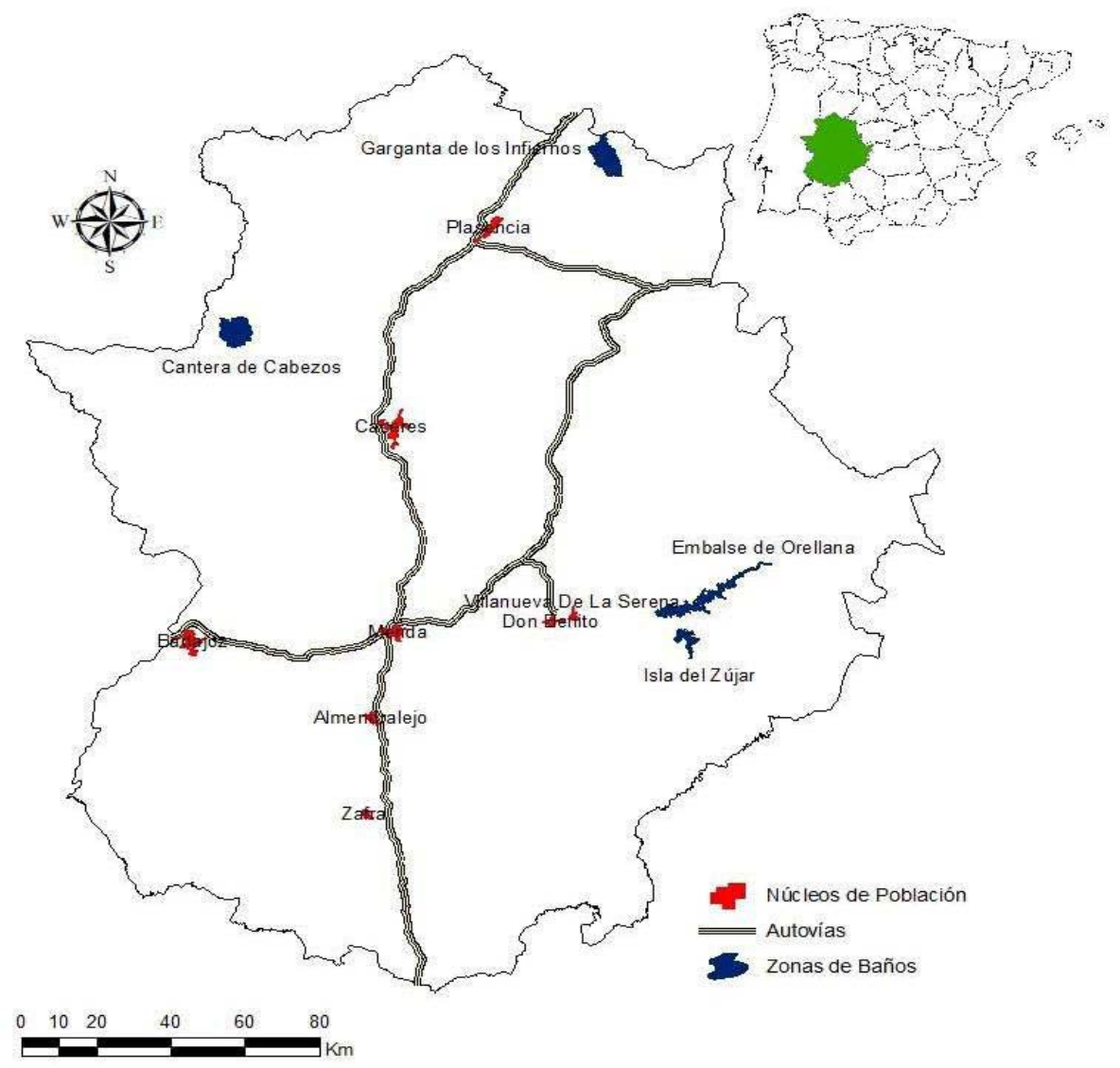

\subsection{Recursos turísticos}

Todos los trabajos han recopilado e identificado los recursos turísticos de las áreas de estudio y de su entorno (a diferentes escalas) a partir de información obtenida a través de páginas webs en Internet, en guías especializadas, haciendo entrevistas telefónicas y personales a agentes clave y mediante trabajo personal de campo. Después de esa recopilación, los recursos turísticos son seleccionados y presentados por categorías y por municipios, en los casos en los que las áreas de baño mantengan una estrecha vinculación con el entorno. En ocasiones, la descripción por municipios responde a criterios de gestión como la presencia de Grupos de Acción Local (GAL's) y los municipios que estos abarcan.

En el caso de Orellana, debido a ser un trabajo centrado principalmente en la oferta turística, la autora ha completado todas las fases. Los recursos turísticos, tras su identifi- 
cación, fueron localizados geográficamente con un GPS de mano, se tomaron fotografías y fueron inventariados y catalogados siguiendo los criterios que establece la Organización de Estados Americanos (OEA) (González Ruiz, 2014). Para ello, se utilizó una serie de formularios utilizables mediante el software Microsoft Office Access, que fueron creados por el Grupo de Turismo de la Diputación Provincial de Cáceres para realizar el Inventario de Recursos Turísticos del Parque Natural del Tajo Internacional en 2010. Finalmente, se hizo un grupo focal con 2 graduados en Turismo y 1 residente en la localidad para establecer criterios objetivos a la hora de puntuar cada uno de los recursos y establecer entre ellos un cierto orden jerárquico de importancia.

\subsection{Oferta turística}

Dependiendo del ámbito geográfico y de la finalidad de cada trabajo, se profundizó a diferentes intensidades en el ámbito de la oferta turística. A grandes rasgos, se intentó recopilar la máxima información posible sobre alojamiento, restauración y oferta complementaria (Varela Merino et al., 2003). Respecto al alojamiento, se hizo distinción entre hoteles, pensiones, apartamentos, casas rurales y campings. En el tema de restauración, se diferenció entre restaurantes, bares y chiringuitos y referente a la actividad complementaria se abordó el tema de las actividades lúdicas que se ofertan o pueden ser ofertadas en cada uno de los sitios.

Mucha de esta información fue obtenida a partir de entrevistas a agentes clave como miembros de la Corporación Municipal del Ayuntamiento de Orellana, población local de los municipios que paseaba por el entorno en el momento del trabajo de campo, el Presidente de la Mancomunidad de la Serena (D. Eduardo Tena Torres), el Gerente del Complejo de Turismo Rural Isla del Zújar (D. Nicolás Peña Paredes), la Técnico en Turismo de la Oficina de Turismo del Valle del Jerte ( $\mathrm{D}^{\mathrm{a}}$ Esperanza Izquierdo Regadera), la Gerente ( $\mathrm{D}^{\mathrm{a}}$ Elisa Esteban Trenado) y la Técnico en Desarrollo Rural ( $\mathrm{D}^{\mathrm{a}}$ Consuelo Sánchez Bardón) de la Sociedad para la Promoción y Desarrollo del Valle del Jerte (SOPRODEVAJE), así como a trabajadores y gerentes de diferentes balnearios y Oficinas Municipales de Turismo. Algunos de ellos no han sido mencionados por expresar su deseo de mantenerse en el anonimato.

En algunos trabajos donde la componente histórica de la oferta, es decir, en qué años se comenzaron a construir los embalses, preparar las playas artificiales, las infraestructuras turísticas planificadas por la administración, los diferentes balnearios, etc., las entrevistas a agentes clave no iban sólo encaminadas a conocer el presente del número de establecimientos o actividades turísticas y sus tipologías, sino que también se centraron en actuaciones pretéritas. Toda esta información fue complementada con trabajo de campo y, en algunos casos, ha sido cartografiada usando aplicaciones informáticas como Google Maps.

\subsection{Demanda turística}

El turismo de baño en España ha sido tradicionalmente contemplado como algo que se hacía en espacios de bordes de agua, ligados únicamente al sol y a la playa (Valero, 1994). Es en este tipo de espacios, y normalmente a escala provincial o autonómica, donde se concentran la mayoría de los trabajos (Baños Castiñeira, 1998; Vera Rebollo y Baños Castiñeira, 2010). 
En este caso, los estudios de demanda turística a escala de enclave, complementado con la entidad territorial local o supramunicipal a la que pertenece, se basaron principalmente en encuestas directas a pie de calle en el lugar de estudio, que se complementaron en algunos casos con los datos suministrados por Oficinas de Turismo o alguna otra entidad.

Las encuestas se hicieron en temporada estival (150 en la Isla del Zújar, 315 en Los Pilones y 216 en la Cantera de Alcántara) centradas en conocer el perfil del visitante (sexo, edad, lugar de procedencia, motivaciones, días y lugar de pernoctaciones, etc.) y en el caso de la Garganta de los Infiernos, conocer también si los veraneantes de Los Pilones sabían de la existencia de otras gargantas o zonas de baño que hay en la Comarca del Valle del Jerte. Los cuestionarios, rellenados in situ por los veraneantes, eran de muy pocas preguntas (no más de 15), sencillas, de carácter breve y con respuestas múltiples cerradas, que han permitido realizar estadística descriptiva básica (e.g. tablas de frecuencia).

En los trabajos de Orellana y sobre el turismo de balnearios en Extremadura, como el objetivo prioritario no era conocer la demanda turística, tan sólo se mencionan datos obtenidos a partir de las entrevistas claves donde, por ejemplo, un empleado del Ayuntamiento de Orellana hace una estimación del número de turistas que visitan los fines de semana la zona de baño a partir de lo que se recauda por la zona azul del aparcamiento. O el Director del Balneario de Baños de Montemayor estima la proporción de turistas que usan su instalación. Esta información se complementó con datos provenientes del Observatorio Nacional del Termalismo.

La información obtenida ha permitido realizar cartografía para ver el área de influencia de cada uno de las áreas de baño aquí analizadas (mapas de isócronas no añadidos al cuerpo de este texto), así como de inferir un patrón de perfil de usuario de este tipo de atractivo turístico (lugar de procedencia, motivación de la visita, grupo de edad, lugar de pernoctación, fuente de información para el conocimiento del recurso, etc.). En el caso de la Garganta de los Infiernos, incluso ha permitido saber cuál es el interés comparativo de Los Pilones con respecto a otras zonas de baño, que si bien no son tan accesibles, al menos, tienen bastante interés propagandístico (e.g. Cascada del Caozo y su pasarela-mirador).

\section{RESULTADOS}

\subsection{Recursos turísticos}

La Tabla 1 resume el número de recursos turísticos que fueron identificados en cada una de las áreas de estudio acorde a las categorías propuestas por la OEA (Navarro, 2015). Además, añade el número de espacios protegidos según el organismo o la administración de la que proviene la figura de protección: Humedal de Importancia Internacional y Reserva de la Biosfera (UNESCO), Zona de Especial Conservación y Zona de Especial Protección de Aves (Red Natura 2000 de la Unión Europea), Red de Espacios Naturales Protegidos de Extremadura (Junta de Extremadura) y Bienes de Interés Cultural (BIC, Gobierno de España y Junta de Extremadura).

Desde un punto de vista natural, sobresalen Los Pilones debido a la red de numerosas gargantas y arroyos que conforman la zona (Muñoz Barco et al., 2014). De hecho, ésta es la razón por la que se llevó a cabo el estudio, es decir, hacer un análisis comparativo 
entre Los Pilones y otras muchas gargantas de la zona aptas para el baño (más detalles en el apartado de Demanda Turística y en la Discusión). En segundo lugar, se encuentra el Embalse de Orellana por sus numerosas rutas, además de su playa, embalse y embarcadero. La Isla del Zújar presumiblemente tiene menos recursos al tratarse de un complejo de turismo rural y la Cantera de Cabezos probablemente los subestime porque ése no era el objetivo principal del trabajo (Andrades Caldito, 2008).

Respecto a los recursos culturales, se han considerado los de la localidad más cercana a la zona de baño, ya que formaría parte de la oferta complementaria cultural que un turista pueda disponer en relativamente poco tiempo. A Orellana la Vieja se la localizaron 11 recursos aunque tan sólo 1 es un Bien de Interés Cultural (Palacio-Castillo del Marqués de Belgida). En Alcántara también se localizaron 11 recursos, de los cuales 4 son BIC (Convento de San Benito, Iglesia de Santa María de Almocóvar, Peña Buraca y el Puente Romano). Cabezuela del Valle también tiene 1 BIC, su conjunto histórico, y la Isla del Zújar se limita a aspectos arquitectónicos de reciente creación (p.ej. Centros de Interpretación) (Muñoz Barco et al., 2014).

La gastronomía, festividades y acontecimientos programados han sido inventariados en mayor cantidad en el caso del Embalse de Orellana al tratarse de un trabajo específico centrado en la oferta turística. No obstante, platos típicos, oficios tradicionales, festividades de carácter religioso y la celebración con cierta periodicidad de eventos culturales y deportivos como la Fiesta del Cerezo en Flor de Interés Turístico Nacional o el Campeonato de Extremadura de Triatlón aportan un gran valor turístico complementario a estas zonas de baño.

Los visitantes de estas zonas de baño estarán también disfrutando de espacios naturales protegidos por la UNESCO (Orellana es un Sitio Ramsar y la Cantera de Cabezos está en una Reserva de la Biosfera), por la Red Natura 2000, sobre todo, por su gran riqueza ornitológica y con figuras de protección autonómicas de gran calado como la Reserva Natural de la Garganta de los Infiernos, el Parque Natural del Tajo Internacional y una serie de Árboles Singulares.

Tabla 1

NÚMERO TOTAL DE RECURSOS TURÍSTICOS IDENTIFICADOS. SE INCLUYE EL NÚMERO DE ESPACIOS NATURALES PROTEGIDOS RECONOCIDOS POR LA UNESCO, LA UNIÓN EUROPEA, EL ESTADO ESPAÑOL O POR LA JUNTA DE EXTREMADURA

\begin{tabular}{lccccccc}
\hline Área & $\begin{array}{c}\text { Sitios } \\
\text { naturales }\end{array}$ & $\begin{array}{c}\text { Sitios } \\
\text { culturales } \\
\text { (BIC) }\end{array}$ & $\begin{array}{c}\text { Folclore } \\
\text { Acontecimientos } \\
\text { programados }\end{array}$ & UNESCO & $\begin{array}{c}\text { Red Natura RENPEX } \\
\mathbf{2 0 0 0}\end{array}$ & \\
\hline $\begin{array}{l}\text { Embalse de } \\
\text { Orellana }\end{array}$ & 9 & 1 & 12 & 1 & 1 & 1 & 0 \\
$\begin{array}{l}\text { Isla del } \\
\begin{array}{l}\text { Zújar } \\
\text { Los Pilones }\end{array}\end{array}$ & 5 & 0 & 1 & 2 & 0 & 0 & 0 \\
$\begin{array}{l}\text { Cantera de } \\
\text { Cabezos }\end{array}$ & 5 & 1 & 5 & 3 & 0 & 1 & 2 \\
\hline
\end{tabular}




\subsection{Oferta turística}

En este apartado, se compara la oferta turística en términos de alojamiento (Figura 2), restauración (Figura 3) y actividades complementarias entre las cuatro zonas de baño (Tabla 2).

Los datos aquí aportados se ciñen a lo recopilado en los trabajos analizados mediante trabajo de campo y no se adaptan necesariamente al ámbito municipal. Podrían haberse añadido datos oficiales de oferta turística, estandarizados según la tipología establecida por la normativa regional, de cada uno de los municipios pero se desestimó esa idea al ir en contra de la filosofía de estos trabajos (búsqueda de información a escalas que no aparecen en las estadísticas oficiales).

En cuanto a la oferta de alojamiento, se observan comportamientos totalmente diferentes entre las áreas de estudio, aunque, en parte, esto puede venir ocasionado por las diferentes formas en que cada uno de los trabajos trató la recopilación de datos. Las localidades próximas del Embalse de Orellana ofrecen alojamiento en hoteles, hostales y pensiones. Lo mismo ocurre con la de Alcántara, con 8 establecimientos de alojamiento, pero no han sido considerados en este trabajo por circunscribirse al ámbito más próximo de la cantera.

El caso del Zújar es bastante peculiar, ya que toda la oferta de alojamiento se concentra en las propias instalaciones del Complejo de Turismo Rural, es decir las 3 casas rurales, los 9 apartamentos y el albergue con 65 plazas. Algo parecido ocurre en los alrededores de La Cantera de Cabezos donde todo el soporte en temas de alojamiento (y restauración) se circunscribe a un camping. Esta última tipología está presente en todas y cada una de las cuatro áreas de baño y son de las tipologías más utilizadas por los bañistas en verano, gracias a su bajo precio y cercanía al recurso más importante.

\section{Figura 2 \\ NÚMERO DE ESTABLECIMIENTOS DE ALOJAMIENTO EN LAS ÁREAS ESTUDIADAS (2016)}

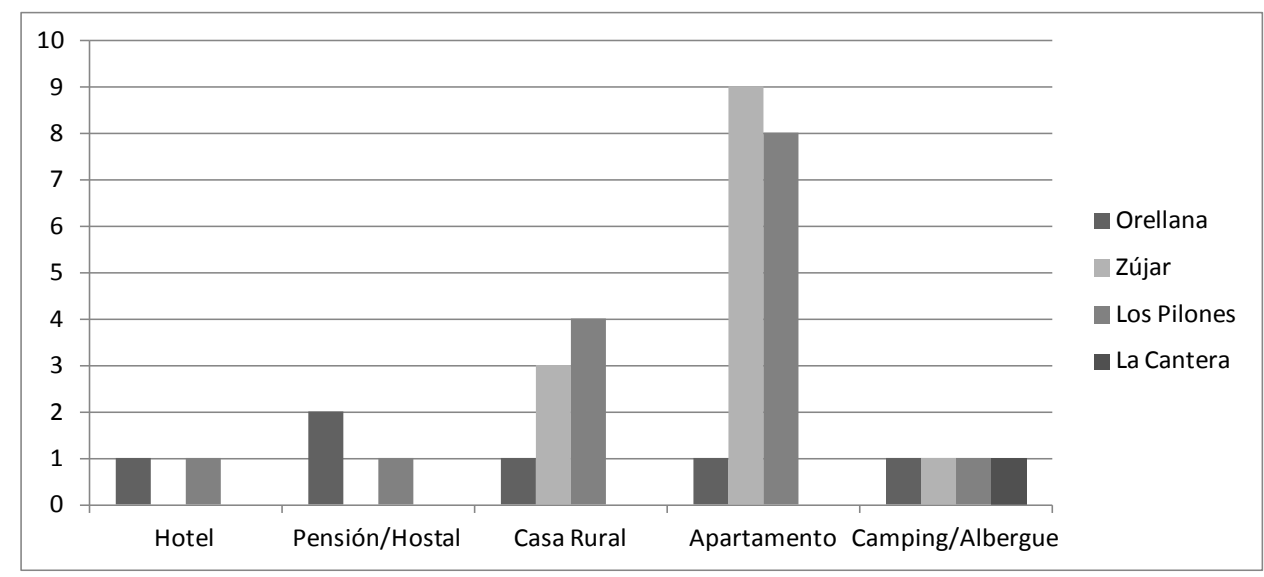

Fuente: Autores de los trabajos. 
La oferta en restauración se ciñe a la presencia de algunos restaurantes, bares o chiringuitos relativamente cercanos a los lugares de baño para dar un servicio más inmediato. Normalmente, se suele tratar de un único restaurante y bar/chiringuito, que, en ocasiones, es incluso gestionado por la misma concesionaria. En el caso de Orellana, el número de restaurantes aumenta porque han sido considerados algunos muy cercanos pero situados ya en la propia localidad. Por lo comentado en algunas de las entrevistas, los fines de semana y festivos de mayor tránsito alrededor de la playa de bandera azul, numerosos bañistas deciden acercarse al pueblo para escapar de la aglomeración contextual. Llamativo, con 3 bares, es el caso de la Isla del Zújar, que responde bien a la necesidad de servicio en momentos puntuales de mayor reclamo.

\section{Figura 3 \\ NÚMERO DE ESTABLECIMIENTOS DE RESTAURACIÓN EN LAS ÁREAS DE BAÑO Y SU ENTORNO FUNCIONAL INMEDIATO (2016)}

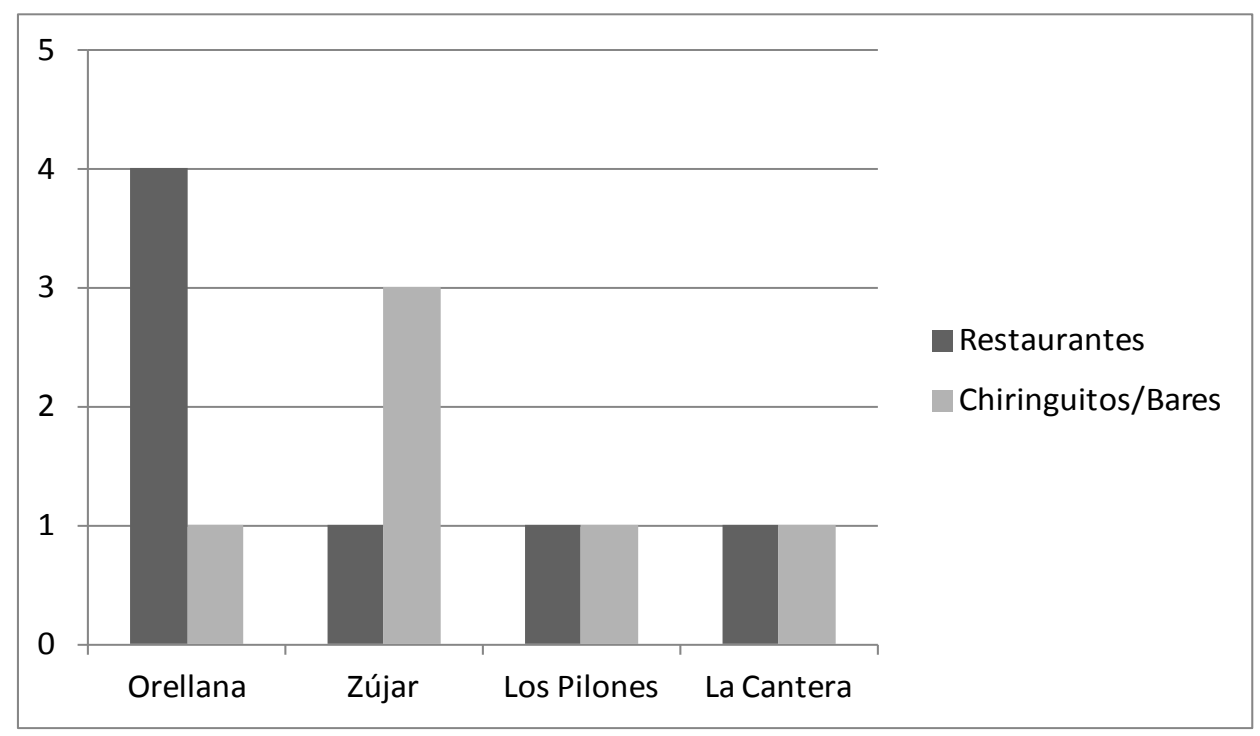

Fuente: Autores de los trabajos.

Respecto a la oferta complementaria, se observan 2 "velocidades" claramente entre los grupos de las zonas de baño. Aquellas localizadas en los embalses están pensadas para ofrecer un gran abanico de actividades a los visitantes durante su estancia, mientras la cantera y la garganta son enclaves naturales protegidos y restringidos que tan sólo ofrecen baño, observación de aves y la realización de rutas. No obstante, cerca de ellas existen grandes embalses y ríos en los que se podría complementar con otras actividades, además de pueblos con oferta cultural y de servicios. 


\section{Tabla 2 \\ OFERTA COMPLEMENTARIA DE LAS ZONAS DE BAÑO ESTUDIADAS}

(2016)

\begin{tabular}{lccccccc} 
Área & Birdwatching & Senderos & $\begin{array}{c}\text { Rutas } \\
\text { Bici }\end{array}$ & Buceo & Navegación & Pesca & $\begin{array}{c}\text { Deportes } \\
\text { náuticos }\end{array}$ \\
\hline Embalse de Orellana & SÍ & SÍ & Sí & SÍ & A motor/ vela & Sí & Sí \\
Isla del Zújar & SÍ & SÍ & SÍ & SÍ & SÍ & Sí & SÍ \\
Los Pilones & SÍ & SÍ & Sí & NO & NO & NO & NO \\
Cantera de Cabezos & SÍ & SÍ & Sí & NO & NO & NO & NO \\
\hline
\end{tabular}

Fuente: Autores de los trabajos.

\subsection{Demanda turística}

Dentro del análisis de la demanda turística, se recopiló información sustancial sobre aspectos como la estimación del número total de visitantes (Figura 4) y su procedencia geográfica (Tabla 3), así como datos aislados de gasto turístico, número de pernoctaciones, modalidades turísticas, conocimiento de otras zonas de baño, etc.

Respecto al número total de personas que visitan cada uno de estos enclaves, no existe ningún recuento fidedigno y, ni tan siquiera, es estudiado rigurosamente por ningún interesado. En el caso de los trabajos aquí recopilados, se recurrió a asunciones o aproximaciones a partir de datos obtenidos por otras instituciones. Según la información suministrada por la secretaria del Ayuntamiento de Orellana, unas 60.000 personas pueden bañarse cada verano en la playa de Orellana. Esa afirmación se basa en una recaudación promedio de 20.000 EUR/año en concepto de pago de zona azul por aparcar los fines de semanas y festivos de verano. El pago es de 1,50 EUR/coche y se asume un promedio de 4 personas por coche, lo que dan un total de 50.000 personas a los que se puede sumar una estimación de otras 10.000 que vienen en autobuses contratados ex proceso.

Para la Isla del Zújar, no existe todavía ningún registro que permita hacer una idea del total de los turistas, pero en el caso de Los Pilones, al menos se sabe que unas 25.000 personas al año pasan por la Oficina Comarcal de Turismo de Cabezuela del Valle (localidad más cercana), y la gran mayoría solo conocen Los Pilones como zona de baño en detrimento de otras gargantas, y en el caso de Alcántara más de 55.000 pasaron por su Oficina Local de Turismo a pedir información turística en el año 2018.

Respecto al gasto turístico, más de la mitad de los encuestados en la Isla del Zújar admitieron tener un presupuesto de 50 EUR para gastar cada día que pasan en el recinto. Y, según las palabras del gerente del complejo, suelen pernoctar aquellas personas cuyas localidades están a una distancia superior a los $100 \mathrm{~km}$ (1 hora de viaje en coche aprox.). El $40 \%$ de las personas encuestadas en la Isla del Zújar venían de una distancia inferior a $\operatorname{los} 50 \mathrm{~km}$, el $25 \%$ entre 50 y $75 \mathrm{~km}$ y tan solo un $10 \%$ a una distancia entre 75 y 100 $\mathrm{km}$. Curiosamente, tan sólo un $12 \%$ de estos turistas expresaron sentirse atraídos por la posibilidad de hacer deportes acuáticos y solo un $12 \%$ dicen que visitan este enclave muy a menudo. 


\section{Figura 4 \\ NÚMERO ESTIMADO DE TURISTAS QUE VISITAN CADA Ã̃O ESTAS ZONAS DE BAÑO}

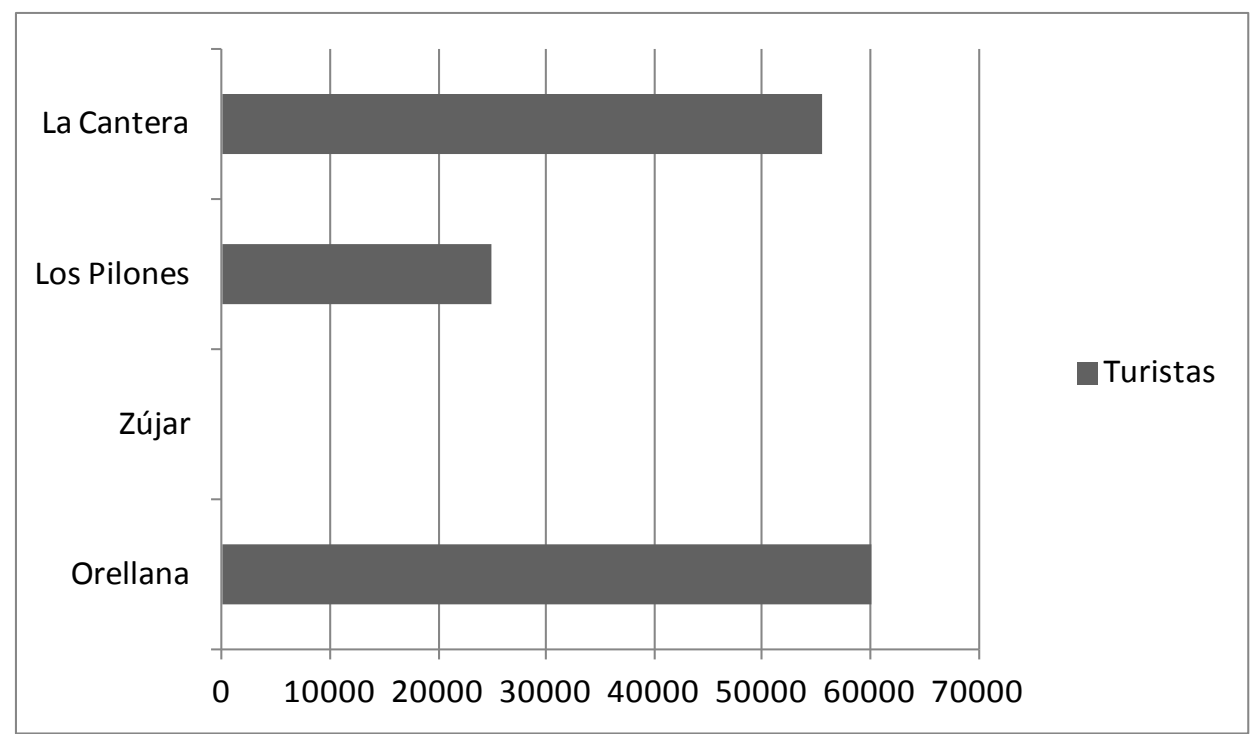

Fuente: Autores de los trabajos.

La procedencia de los turistas a estos destinos de baño es mayoritariamente extremeña y, en muchos casos, de personas de la propia comarca. Con respecto al porcentaje restante que, a grandes rasgos, son de otras partes de España, en realidad, se trata de diferentes fórmulas de turismo paisano donde emigrantes, hijos o nietos de personas que emigraron se encuentran de vacaciones en el lugar con el que mantienen su vínculo emocional, que puede estar cerca de estas áreas de baño, en muchos casos. No se encuestó a ninguna persona extranjera pero sí consta en las Oficinas Municipales y Comarcales de Turismo el interés de ellos por esta zona de baño. Sorprende la ausencia de turismo portugués en la Cantera de Cabezos.

Tabla 3

\section{PROCEDENCIA GEOGRÁFICA DE LOS TURISTAS (EN PORCENTAJE)}

\begin{tabular}{lcccc} 
Área & Comarca & Extremadura & Otras regiones & Extranjeros \\
\hline Embalse de & n.d. & n.d. & n.d. & n.d. \\
Orellana & & & & \\
Isla del Zújar & 50 & 75 & 25 & 0 \\
Los Pilones & n.d. & 59 & 41 & 0 \\
Cantera de & n.d. & 35 & 65 & 0 \\
Cabezos & & & & \\
\hline
\end{tabular}

Fuente: Autores de los trabajos. 


\section{DISCUSIÓN}

La política de construcción de embalses del siglo XX en Extremadura, que inicialmente se pensó para la agricultura de irrigación y el aprovechamiento hidroeléctrico, ha generado una nueva serie de usos, de los cuales el recreativo, particularmente, en verano parece estar teniendo muy buena aceptación por un amplio espectro de la sociedad: turistas, población local, políticos, conservacionistas, emprendedores, etc. La consecución de la bandera azul por parte de Orellana en 2010 y de Cheles en 2016, junto a otras muchas iniciativas que fomentan el turismo de sol y playa en esta costa interior, han hecho posible que, hoy en día, el turismo de baño sea una modalidad turística muy a tener en cuenta por la sociedad extremeña.

Este turismo de baño, con una marcada estacionalidad estival, quizás pueda ser complementado por el fenómeno reciente del birdwatching, ya que los embalses suponen un recurso esencial para muchas aves migratorias y eso atrae a muchos turistas, sobre todo, del resto de Europa. La posibilidad de practicar deportes náuticos y la relativa cercanía a Madrid están haciendo de estas zonas de costa interior un lugar apetecible para el desarrollo de infraestructuras turísticas. No obstante, el mediático caso del derribo del Complejo Marina Valdecañas por un problema de urbanización en suelo protegido por la Red Natura 2000 puede ser un gran fenómeno disuasorio en este sentido, sin que esto deba ser interpretado como un comentario crítico a ninguna de las sentencias judiciales que se han dictado.

No obstante, a la hora de realizar este trabajo, se siguen detectando carencias que dificultan poder llevar políticas certeras en este sentido. A fecha de hoy, el turismo de baño en Extremadura sigue siendo un fenómeno poco estudiado por la comunidad investigadora, y sigue habiendo mucho terreno por avanzar en lo que a la promoción y formación turística se refiere. También se detecta una desconexión entre los recursos naturales y culturales del entorno con la práctica del baño, siendo en muchos casos una modalidad turística muy básica de baño, comida y refrigerio en restaurantes y bares próximos y, en ocasiones, alguna actividad complementaria de esparcimiento: motos náuticas, pedaletas, etc.

Otro aspecto a tener en cuenta es la presencia en la región de varios lugares de baño con semejantes características: Orellana, Isla del Zújar, Alqueva, Medellín, Cíjara y García Sola. Esto hace, por un lado, la posibilidad de establecer sinergias pero está sirviendo también como un fenómeno de competencia entre destinos, que podría llegar a ocasionar la desaparición o descuido de aquellos más débiles. De hecho, la playa de Orellana y la isla del Zújar, debido a sus $16 \mathrm{~km}$ de distancia y a la cercanía de dos zonas emisoras de bañistas como Don Benito y Villanueva, pueden ser interpretadas como competidoras dentro del mismo mercado. A pesar de que el Zújar recibe menos bañistas, la oferta de alojamiento es mucho más diversificada y completa.

Las gargantas del norte de Extremadura, por su parte, presentan una modalidad turística completamente diferente, ya que en ellas su relativa inaccesibilidad y su notable estado de naturalidad son los principales reclamos turísticos. No obstante, es curioso como más de la mitad de las personas que se encuestaron a pie de campo en Los Pilones solamente conocen esa garganta como posible zona de baño. Podría decirse que la espectacularidad del entorno está eclipsando el resto de zonas de baño y eso puede llegar a causar problemas de superación de la capacidad de carga ecológica y hacer que en el futuro las autoridades de la Reserva Natural deban regular el acceso los fines de semana de verano. 
En el caso del Embalse de Alcántara, la adecuación para el baño de la Cantera de Cabezos ha sido un gran soplo de aire fresco en las posibilidades turísticas de la zona. Es comúnmente conocido por los extremeños el enclave por su singularidad, sin embargo, todavía adolece de poca promoción turística y puede resultar difícil atraer bañistas de la parte portuguesa ya que al otro lado de la frontera existen piscinas naturales (p.ej. Penha Garcia) que son de gran interés para los veraneantes de ambos lados de la raya.

\section{CONCLUSIÓN}

Este análisis es un buen ejemplo de la cantidad de información aprovechable, que nuestros estudiantes de fin de año llegan a recopilar en sus trabajos, y que, desafortunadamente, se mantiene totalmente inédita para la comunidad científica porque no llegan a ser publicados, ni siquiera, en los repositorios de la propia Universidad. En ellos, se ha evidenciado la importancia que tienen las zonas de baño para Extremadura, al menos como atractivo turístico para los habitantes de la región, o con vínculo emocional o personal hacia ella (turismo paisano), y la necesidad de seguir profundizando en su investigación. A pesar de que se ha constatado el predominio de procedencia extremeña en los bañistas, se observan ciertas iniciativas de corte empresarial, bien sea mediante la construcción de complejos o aportando una serie de servicios de alojamiento y de restauración in situ o en las localidades vecinas, que hacen entrever que se está trabajando en una dirección correcta por parte de las administraciones y entidades privadas. Este trabajo ha de servir también como toque de atención, en primer lugar, para los investigadores de la propia casa con el ánimo de que sigan investigando en la línea del turismo de baño, mediante proyectos o coordinando estudiantes entre diferentes profesores, y, por otro, para que la Universidad se replantee su política de publicación de trabajos fin de estudios hacia una total universalización. Por último, pero no por ello menos importante, todo lo expuesto en este trabajo debe ser interpretado con cautela ya que existen numerosas limitaciones intrínsecas de investigación en los estudios analizados.

\section{Agradecimientos}

Este trabajo nunca habría sido posible sin los TFG's de nuestros estudiantes, todos ellos mencionados en las referencias bibliográficas, que sirvieron como fuente de información e inspiración, y que dejan como legado una fructífera relación simbiótica de aprendizaje, colaboración, respeto y perdurable afecto entre todos nosotros. Los autores, además, agradecen la labor del editor y de los evaluadores anónimos, que con sus comentarios han mejorado considerablemente la calidad del manuscrito.

\section{REFERENCIAS BIBLIOGRÁFICAS}

ABEL SCHAAD, D., HERNÁNDEZ CARRETERO, A.M., LÓPEZ-MERINO, L., PULIDO DÍAZ, F.J. y LÓPEZ SÁEZ, J.A. (2009): «Cabras y quemorros: tres siglos de cambios en el paisaje de la vertiente extremeña de la Sierra de Gredos», Revista de Estudios Extremeños, ${ }^{\circ}$ LXV, pp. 449-478. 
ALONSO DE LA TORRE, J.R. (2019): «El país de las 72 gargantas», Diario Hoy, Fecha de publicación: 15 junio 2019.

ANDRADES CALDITO, L. (2008): «Planificación turística y sostenible. Aplicación a un destino de costa interior de Extremadura: El embalse de La Serena», Revista de Estudios Empresariales. Segunda época, nº 2, pp. 24-47.

BAÑOS CASTIÑEIRA, C.J. (1998): «La oferta turística complementaria en los destinos turísticos alicantinos: implicaciones territoriales y opciones de diversificación», Investigaciones Geográficas, $\mathrm{n}^{\circ} 19$, pp. 85-103.

CAMPESINO FERNÁNDEZ, A.J. (2016): «Paisajes del agua y turismo fluvial en la Raya ibérica», en Paisaje, cultura territorial y vivencia de la Geografía. Libro homenaje al profesor Alfredo Morales Gil. San Vicente del Raspeig, Publicaciones de la Universidad de Alicante, pp. 47-72.

CEBRIÁN ABELLÁN, F. y GARCÍA GONZÁLEZ, J.A. (2010): « Propuesta metodológica para la identificación, clasificación y puesta en valor de los recursos territoriales del turismo interior. La provincia de Albacete», Boletín de la Asociación de Geógrafos Españoles, $\mathrm{n}^{\circ}$ 54, pp. 361-383.

DIEZ, L., RAMALLO, S y ULLOA, C. (2019): Turismo exterior en España: Pérdida de tracción a la espera de mejoras en competitividad. Observatorio Económico, BBVA Research.

DIRECCIÓN GENERAL DE TURISMO (2010): Plan Estratégico de Turismo para Extremadura 2010-2015. Mérida, España, Consejería de Cultura y Turismo, Junta de Extremadura.

FERNÁNDEZ BARROSO, J. (2020): Análisis exploratorio de La Cantera de Alcántara como principal zona de baño de la Comarca Tajo-Salor-Almonte. TFG de Turismo, Universidad de Extremadura.

GARCÍA GONZÁLEZ, L. (1991): «El sector turístico en el desarrollo de Extremadura», Alcántara: Revista del Seminario de Estudios Cacereños, $\mathrm{n}^{\circ} 22$, pp. 347-378.

GARCÍA GONZÁLEZ, L. (2004): «Agua y turismo. Nuevos usos de los recursos hídricos en la Península Ibérica. Enfoque integral», Boletín de la Asociación de Geógrafos Españoles, $\mathrm{n}^{\circ}$ 37, pp. 239-255.

GÓMEZ SÁNCHEZ, S. (2016): Análisis comparativo del interés turístico de la Garganta de los Infiernos. TFG de Turismo, Universidad de Extremadura.

GONZÁLEZ RUIZ, T. (2014): Distribución de los recursos culturales en España y su relación con el turismo cultural. Málaga, España. TFG de Turismo, Universidad de Málaga.

JUNTA DE EXTREMADURA (2016): Plan Turístico de Extremadura 2017-2020. Mérida, Dirección General de Turismo.

LECO BERROCAL, F., MATEOS RODRÍGUEZ, A.B. y PÉREZ DÍAZ, A. (2015): «Estudio de la demanda del turismo en el Parque Nacional y Reserva de la Biosfera de Monfragüe», Cuadernos de Turismo, n 35, pp. 231-257.

LÓPEZ OLIVARES, D. (2001): « La evaluación de los recursos territoriales turísticos de las comarcas del interior castellonense (Comunidad Valenciana)», Investigaciones Geográficas, $\mathrm{n}^{\circ} 25$, pp. 137-157.

MATAMOROS CODER, P. y CARRASCOSA MOLINER, B. (2014): «La situación del patrimonio arqueológico subacuático en la cuenca extremeña del Tajo. Perspectivas de conservación, documentación y análisis», en Arqueología subacuática española: Actas 
del I Congreso de Arqueología Naútica y Subacuática Española, Cartagena, 14, 15 y 16 de marzo de 2013. UCA Editores, pp. 67-80.

MUÑOZ BARCO, P., MARTÍNEZ FLORES, E y GARZÓN HEYDT, G. (2014): «Patrimonio geológico de Extremadura y conservación del paisaje», Geogaceta, n ${ }^{\circ}$ 55, pp. 47-50. MUÑOZ BARCO, P., REBOLLADA CASADO, E., TENA REY, M.T. y CUBERO, J.J: (2014): «Catálogo de marmitas de gigante de Extremadura», en XIII Reunión Nacional de Geomorfología. Cáceres, Sociedad Española de Geomorfología, pp. 123-126.

NAVARRO, D. (2015): «Recursos turísticos y atractivos turísticos: conceptualización, clasificación y valoración», Cuadernos de Turismo, n 35, pp. 335-357.

NIETO MASOT, A. y CÁRDENAS ALONSO, G. (2017): «25 Años de políticas europeas en Extremadura: turismo rural y método LEADER», Cuadernos de Turismo, n 39 , pp. 389-416.

PINO-DEL-CARPIO, A., ARIÑO, A.H., VILLARROYA, A., PUIG, J. y MIRANDA, R. (2014): «The biodiversity data knowledge gap: Assessing information loss in the management of Biosphere Reserves», Biological Conservation, $\mathrm{n}^{\circ}$ 173, pp. 74-79.

REBOLLADA CASADO, E., CAMPOS RODAS, M.A y CABEZAS HERNÁNDEZ, M.T. (2016): «Estado del arte del termalismo en Extremadura: Recursos minero-medicinales, condicionantes ambientales, tramitación administrativa y aprovechamiento turístico y terapéutico», en Derecho ambiental en tiempo de crisis: comunicaciones presentadas al Congreso de la Red ECOVER (A Coruña, 19 y 20 de noviembre de 2015). Tirant lo Blanch, pp. 257-288.

ROBINA RAMÍREZ, R. y PULIDO FERNÁNDEZ, M. (2018): «Religious Experiences of Travellers Visiting the Royal Monastery of Santa María de Guadalupe (Spain)», Sustainability, $\mathrm{n}^{\circ} 10$, pp. 1890.

SÁNCHEZ LOMBA, F. M. (2014): «Salud, patrimonio y agua en la cuenca cacereña del tajo», en Patrimonio cultural vinculado con el agua: paisaje, urbanismo, arte, ingenieria y turismo. Editora Regional de Extremadura, pp. 247-264.

SANTOS REDONDO, E. (2018): Análisis de la oferta y demanda turística de la Isla del Zújar. TFG de Turismo, Universidad de Extremadura.

VALERO, A. (1994): «El turismo de playa en España entre 1850 y 1950 (creación, madurez y crisis)», en Desarrollo regional y crisis del turismo en Andalucía: Actas del simposio hispano-francés: Almería 25-29 de junio, 1991. Instituto de Estudios Almerienses, pp. 297-329.

VARELA MERINO, B., LÓPEZ LAGO, A. y MARTÍNEZ SERRANO, A. (2003): «Primeras aproximaciones al estudio estadístico del alojamiento privado con fines turísticos desde una perspectiva de oferta», Estudios Turísticos, no 155-156, pp. 87-112.

VÁZQUEZ WIZNER, S. (2017): Análisis de la oferta turística del Embalse de Orellana. TFG de Turismo, Universidad de Extremadura.

VERA REBOLLO, J.F. y BAÑOS CASTIÑEIRA, C.J. (2010): «Renovación y reestructuración de los destinos turísticos consolidados del litoral: las prácticas recreativas en la evolución del espacio turístico», Boletín de la Asociación de Geógrafos Españoles, $\mathrm{n}^{\circ} 53$, pp. 329-353.

VIANA CARDADOR, M.I. (2018): Los balnearios de Extremadura: Un recurso turístico infrautilizado. TFG de Turismo, Universidad de Extremadura. 